\title{
Unconventional Magnetic Behavior in Multicomponent Compounds
}

Final Report on DE-FG03-01ER45876 for the Period 2/1/01 through 1/31/04

Date of Report: December 2004

Author: Warren E. Pickett

Originating Research Organization: DOE Office of Science

Information Category: Final Report

Distribution Statement: Unrestricted 


\section{A Results from This Project}

This funding supported investigations of the behavior of complex materials. Very often the chosen materials were structurally complex, since in such cases more and new degrees of freedom that lead to more complicated interactions and more variable behavior. We summarize the most successful parts of that research.

\section{A.1 Calculation of Wannier Functions, and Application to Magnetic Insulators}

Under our prior funding, we initiated and made strong progress, with postdoc Wei Ku (now a staff member at Brookhaven National Laboratory) doing the calculations, on the computation of Wannier functions (WFs), following in a general way the prescription of Marzari and Vanderbilt[1] for constructing maximally localized Wannier functions. Maximal localization has not been pursued specifically in our work, however, since there is no formal reason to do so, and other considerations seem more pressing (see Sec. C). Our procedure was coded for an all-electron, full-potential method (the demanding WIEN97 LAPW code[2]) and the Wannier functions were found to be rich in structure. An illuminating (and representative) example is shown in Fig. 1 Note that, in an effectively single band systems such at CCTO in Fig. 1, there is no question of what atomic character(s) must be included in the WF, since the WFs must reproduce the same density as the (Bloch) band does.

The calculated WFs for $\mathrm{La}_{4} \mathrm{Ba}_{2} \mathrm{Cu}_{2} \mathrm{O}_{10}$ (La422) has enabled us to identify the microscopic mechanisms of this puzzling insulating ferromagnet. $[3,4]$ [There are very few known insulating ferromagnets.] The dominant magnetic coupling, revealed by evaluating the parameters $t, U, J$ for many neighbors (because of the extent of the WF), turns out to be intersite direct exchange, a mechanism that overwhelms the antiferromagnetic superexchange but that had not previously been considered. By contrast, the isostructural Nd422, observed to be antiferromagnetic, has sufficiently different WFs to explain the difference. Two of the main (and surprising) results are: (1) the in-plane order of both La422 and $\mathrm{Nd} 422$ is not controlled by coupling between nearest neighbors, but by a more circuitous route, and (2) the tails of the WFs, lying on the La (Nd) atoms, are very important. Perhaps this should not have been so unexpected: the large observed hyperfine field on the La ion indicated there was induced magnetism on this site (and we showed it is through the formally unoccupied La $5 d$ orbital). Our ability to determine the exchange couplings has led to the identification of $\mathrm{Li}_{2} \mathrm{VOSiO}_{4}$ as the first example of a " $\mathrm{J}_{1}-\mathrm{J}_{2}$ spin system" with $J_{2}>J_{1} \cdot[5,6]$ Our interpretation of magnetic interactions has required a reformulation of the many body Hamiltonian in terms of localized functions (for us, WFs). A more precise specification of this reformulation will get attention in future work.

\section{A.2 Pressure-Induced Transitions in LiH: a Quasiparticle Study}

An opportunity arose to invite Sebastien Lebegue (student of M. Alouani, Strasbourg) to carry out some GW calculations in our group. It was however realized early on that our initial hope, of adapting the codes to spin polarized systems, would take more time than the four months that were available, so the following innovative problem was addressed.

Lithium hydride must be the simplest compound in existence: a strongly ionic crystal with four electrons per unit cell (including core!) and crystallizing in the rocksalt (B1) structure. The Li gives up its valence electron to $\mathrm{H}$, resulting in two ions with the cell, each with $1 s^{2}$ configurations. 

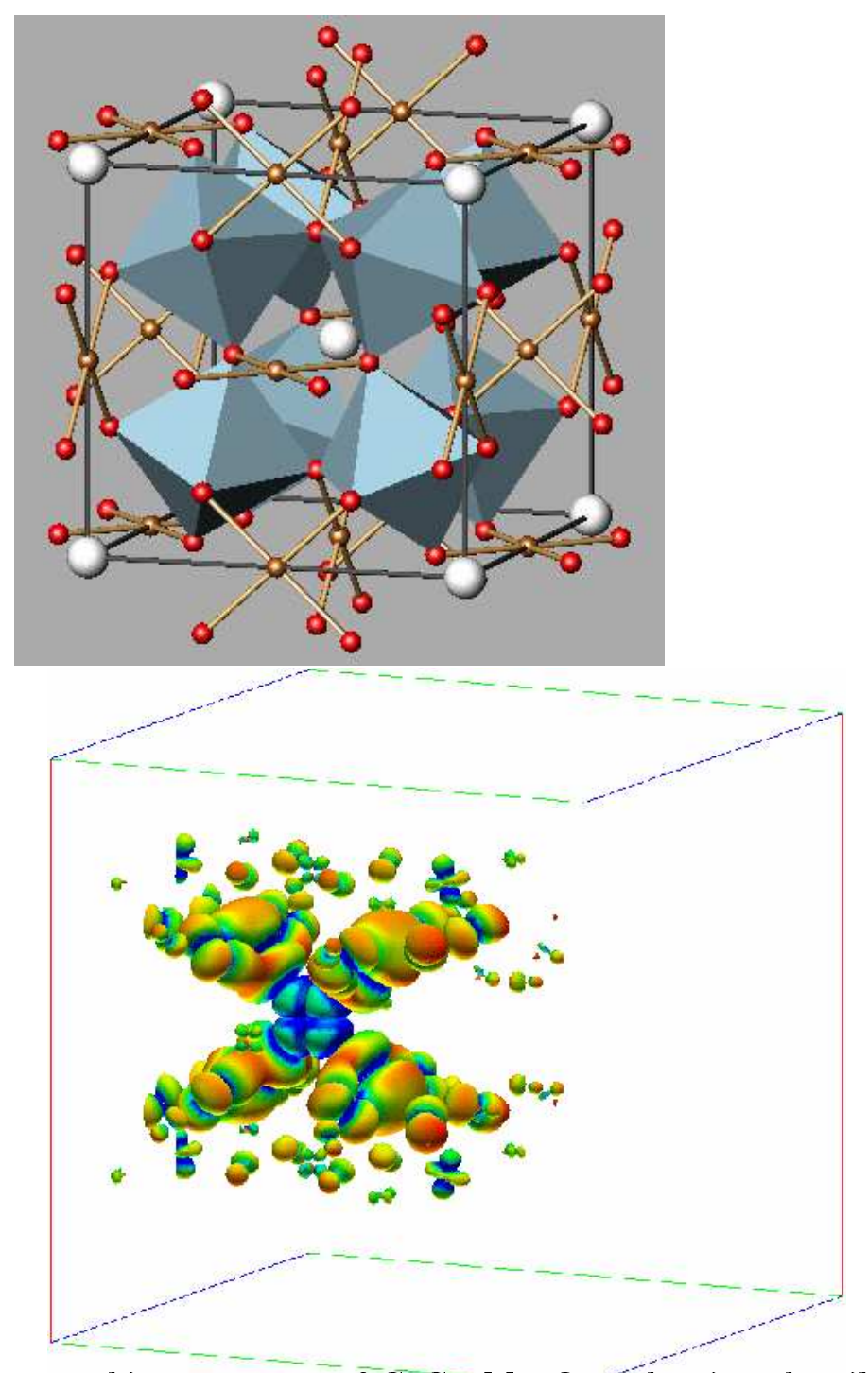

Figure 1: Top: Quadruple perovskite structure of $\mathrm{CaCu}_{3} \mathrm{Mn}_{4} \mathrm{O}_{12}$, showing the tilted $\mathrm{TiO}_{6}$ octahedra (the Ti ion is inside) and the $\mathrm{CuO}_{4}$ plaquettes, oriented perpendicular to $\hat{x}, \hat{y}$, and $\hat{z}$ axes. White balls represent the Ca ions. Each $\mathrm{O}^{2-}$ ion is coordinated with one $\mathrm{Cu}^{2+}$ ion and one $\mathrm{Ti}^{4+}$ ion. Bottom: The $\mathrm{Cu} d_{x z}$-related Wannier function in $\mathrm{CaCu}_{3} \mathrm{Mn}_{4} \mathrm{O}_{12}$, calculated in our group in the prior funding cycle. The $\mathrm{Cu} d_{x z}$ orbital is evident at the center, along with the $d p \sigma$ (anti)bonding to neighboring oxygen $p \sigma$ orbitals (the largest lobes in the figure. $p$ orbitals with smaller amplitude on further oxygens can be identified, along with $d_{3 z^{2}-r^{2}}$ like orbitals (with respect to a local $\hat{z}$ axis) on nominally $d^{0} \mathrm{Ti}$ ions. This compound has three $\mathrm{Cu}$ atoms per (paramagnetic) unit cell, so there are symmetrically related orbitals on two other $\mathrm{Cu}$ ions, oriented along the other cubic axes. The contributions to the WFs from third, fourth, fifth,... atomic neighbors have been found to be important in understanding exchange couplings in this material and in related magnetic insulators. 
Despite this simplicity, $\mathrm{LiH}$ and its isotopes have attracted much attention (experiment and theory) to the study of its properties, e.g., electronic structure, lattice vibrations, behavior under pressure, and defect properties. In addition, possible technological applications have motivated extensive studies in the past, as reviewed by Islam[7]. In particular, the metal-insulator transition (MIT) has been studied by numerous groups. In some alkali hydrides a structural phase transition from the B1 phase to the $\mathrm{B} 2(\mathrm{CsCl})$ phase has been observed[7] and investigated theoretically within the localdensity approximation (see our paper[8] for references). It is only recently that a metal-insulator and structural transition was reported for $\mathrm{LiH}$ at high pressure, and we believe our calculations (combination of ground and excited state) reveal what is really occurring.

We predict a pressure-induced simultaneous metal-insulator and structural transformation (B1 $\rightarrow$ B2 phase) with a $1.3 \%$ volume collapse, using generalized-gradient approximation (GGA) results in conjunction with an all-electron GW approximation method.[8] The local density approximation wrongly predicts that the MIT occurs before the structural phase transition, whereas the GGA predicts the correct transition but with an incorrectly small band gap of the B1 phase at the transition. We have also found, for this compound with only small-mass atoms, that the use of the GGA together with the zero-point vibration correction produces an equilibrium lattice parameter, bulk modulus, and an equation of state that are in excellent agreement with experiment.

\section{A.3 Time-dependent Density Functional Theory: $\mathrm{MgB}_{2}$}

The newly discovered superconductor $\mathrm{MgB}_{2}$ has given us the opportunity to pursue study of dynamical behavior in solids in the context of a exciting new development in materials properties. This study is important (a) scientifically, because $\mathrm{MgB}_{2}$ superconducts at nearly twice as high a temperature $(40 \mathrm{~K})$ as the previous intermetallics, and it does so without $d$ electrons, without a high Fermi level density of states, and without cubic symmetry, all previously thought to be requirements; and (b) for this grant, as preparation for building theories (or models) for the excited states of more highly correlated systems. Under other funding we have made extensive studies of the electronic structure, vibrational properties, and electron-phonon coupling of $\mathrm{MgB}_{2}$ and related materials. Under this funding, we evaluated the response function $\epsilon(\vec{q}, \omega)$ within the framework of time-dependent density functional theory, using (like essentially all such studies so far) the adiabatic approximation for the exchange-correlation functional that arises in the integral equations for the density response. $[9,10]$ The most interesting result was the unexpected discovery of a new, low energy plasmon-like collective mode in the optical response at $\hbar \omega=2.5 \mathrm{eV}$ for $\vec{q}$ along $\hat{c}$ and not too large. This very sharp plasmon contains very little spectral weight compared to the conventional plasmon at $20 \mathrm{eV}$ (also calculated). What it does do is to decrease the plasma edge in the reflectivity from 15-18 eV down to $\sim 2.3 \mathrm{eV}$ (see discussion below). The origin of the new plasmon was traced to the combination of a "pseudogap" in $\epsilon_{2}$ below an onset of strong transitions at $5 \mathrm{eV}$ between $p_{z}(\pi)$ states in the $\mathrm{B}_{2}$ layer and interstitial states with their density mostly in the "Mg layer." This plasmon can be thought of as due to (virtual) charge oscillation between these two sublayers of the compound - a sort of optic plasmon, but at low frequency rather than high frequency.

In the following bibliography, References $3,4,5,6,8,9$, and 10 were supported by this project. 


\section{B Bibliography}

[1] Maximally Localized Generalized Wannier Functions for Composite Energy Bands. N. Marzari and D. Vanderbilt, Phys. Rev. B 56, 12847 (1997).

[2] P. Blaha, K. Schwarz, and J. Luitz, WIEN97, Vienna University of Technology, 1997. Improved and updated version of the original copyrighted WIEN code, which was published by P. Blaha, K. Schwarz, P. Sorantin, and S. B. Trickey, Comput. Phys. Commun. 59, 399 (1990).

[3] Insulating Ferromagnetism in $\mathrm{La}_{4} \mathrm{Ba}_{2} \mathrm{Cu}_{2} \mathrm{O}_{10}$ : an Ab Initio Wannier Function Analysis. W. Ku, H. Rosner, W. E. Pickett, and R. T. Scalettar, Phys. Rev. Lett. 88, 167204 (2002).

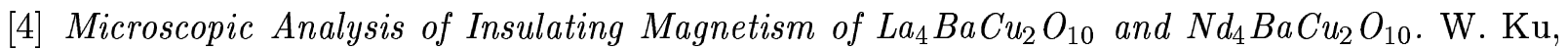
H. Rosner, W. E. Pickett, and R. T. Scalettar, J. Solid State Chem. 171, 329-333 (2003).

[5] High Temperature Expansions for the $J_{1}-J_{2}$ Heisenberg Model: Applications to ab initio Calculated Models for $\mathrm{Li}_{2} \mathrm{VOSiO}_{4}$ and $\mathrm{Li}_{2} \mathrm{VOGeO}_{4}$. H. Rosner, R. R. P. Singh, W. H. Zheng, J. Oitmaa, and W. E. Pickett, Phys. Rev. B 67, 014416 (2002).

[6] Magnetic Hamiltonians for $\mathrm{Li}_{2} \mathrm{VOSiO}_{4}$ and $\mathrm{Li}_{2} \mathrm{VOGeO}_{4}$. H. Rosner, R. R. P. Singh, W. E. Pickett, W. H. Zheng, and J. Oitmaa, Acta Phys. Polonica B 34, 1505 (2003).

[7] A. Islam, Phys. Stat. Sol. B 180, 9 (1993).

[8] Pressure-Induced Simultaneous Metal-Insulator and Structural Phase Transitions in LiH: a Quasiparticle Study. S. Lebegue, B. Arnaud, M. Alouani, and W. E. Pickett, Europhys. Letters 63, 562 (2003).

[9] Ab Initio Investigation of Collective Charge Excitations in $M g B_{2}$. W. Ku, W. E. Pickett, R. T. Scalettar, and A. G. Eguiluz, Phys. Rev. Lett. 88, 027001 (2002).

[10] $\mathrm{MgB}_{2}$ : Complex Behavior from a Simple Compound. H. Rosner, J. M. An, M. Johannes, W. Ku, R. T. Scalettar, W. E. Pickett, S. V. Shulga, S.-L. Drechsler, H. Eschrig, W. Weber, and A. G. Eguiluz, in Studies of High Temperature Superconductors, Vol. 38, edited by A. Narlikar (Nova, New York, 2001). 